\title{
Structural, Magnetic and Dielectric Studies on Strontium Substituted $\mathrm{Nd}_{2} \mathrm{CuO}_{4}$ System
}

\author{
Venugopalan Anbarasu, Appasamy Manigandan, Kandasamy Sivakumar*
}

Department of Physics, Anna University Chennai, Chennai, India.

Email: ksivakumar@annauniv.edu

Received March $7^{\text {th }}, 2010$; revised April 23 ${ }^{\text {rd }}, 2010$; accepted May $15^{\text {th }}, 2010$.

\begin{abstract}
The substitution of Strontium on $T^{\prime}$-structured $\mathrm{Nd}_{2} \mathrm{CuO}_{4}$ system has been carried out through solid state reaction technique. From the Powder XRD patterns, it is found that the compounds are formed in single phase and crystallizes in orthorhombic structure. The variation in lattice parameters with decreasing nature of volume of the prepared compounds confirms the incorporation of lower atomic radii Strontium in Neodymium site. Surface morphology and elemental composition studies are also carried out to know the nature of the compounds and effect of Strontium substitution in $\mathrm{Nd}_{2} \mathrm{CuO}_{4}$ system. The paramagnetic nature of all the prepared compounds has been identified through magnetization studies and the results are correlated with the electron spin resonance studies by the way of variation in resonance field and broad peak width. Increasing order of dielectric constant on higher doping concentration of Strontium and the least value of dielectric loss at higher frequencies confirms the improved surface transport properties of the prepared compounds.
\end{abstract}

Keywords: $\mathrm{Ln}_{2} \mathrm{CuO}_{4}$, Structural Analysis, Powder XRD, Dielectric Studies, Magnetization, ESR Analysis

\section{Introduction}

The $\mathrm{Ln}_{2} \mathrm{CuO}_{4}$ oxides exhibit two different crystal structures with respect to the size of the $\mathrm{Ln}^{3+}$ ion ( $\mathrm{Ln}$, Lanthanide element) that differ mainly in the respective coordination number of cations. In the $\mathrm{Nd}_{2} \mathrm{CuO}_{4}$ system, two types of crystal structures are observed namely $\mathrm{T} / \mathrm{O}$ and $\mathrm{T}^{\prime}$. The occurrence of superconductivity in the $\mathrm{Ln}_{2-\mathrm{x}} \mathrm{Ce}_{\mathrm{x}} \mathrm{CuO}_{4-\mathrm{y}}(\mathrm{Ln}=\mathrm{Nd}, \mathrm{Pr}$ and $\mathrm{Sm})$ family gives a new prospect for understanding the attractive forces as the valence of the Ce dopant suggests that the superconducting carriers are electrons rather than holes. In most high temperature superconducting compounds, $\mathrm{CuO}_{2}$ layers are the fundamental structural units, in which the charge carriers responsible for superconductivity are localized. It is known that electron correlations play an important role in determining the physical properties of these materials. These correlations manifest themselves as two dimensional magnetic fluctuations, which are due to a strong super exchange interaction within the $\mathrm{CuO}_{2}$ layers.

The magnetic properties of the $\mathrm{Ln}_{2} \mathrm{CuO}_{4}(\mathrm{Ln}=\mathrm{Nd}$, Pr, $\mathrm{Eu} \& \mathrm{Gd}$ ) compounds have attracted considerable interest since the discovery of high-temperature superconductivity in these compounds when doped by $\mathrm{Ce}[1,2]$. These compounds having tetragonal type structure $\left(T^{\prime}\right)$, in which the $\mathrm{Ln}^{3+}$ and $\mathrm{Cu}^{2+}$ ions are in eightfold and fourfold coordination respectively $[3,4]$. The structure of compounds in the $\mathrm{Ln}_{2} \mathrm{CuO}_{4}$ (where $\mathrm{Ln}=\mathrm{Nd}, \mathrm{Pr}, \mathrm{Eu}, \mathrm{Sm}$ ) system is similar to the compounds in the La based copper oxides [5]. The main difference arises due to the positions of the oxygen atoms, giving rise to an $\mathrm{O}_{2}$ layer instead of the $\mathrm{La}-\mathrm{O}$ layer. The most important feature of $\mathrm{Nd}$ based materials derives the fact that superconductivity in this system is by electron conduction or n-type rather than hole conduction as in La based superconductors. The electron conducting (n-type) compound $\mathrm{Nd}_{1.85}$ $\mathrm{Ce}_{0.15} \mathrm{CuO}_{4-\mathrm{y}}$ with $\mathrm{T}_{\mathrm{C}}=24 \mathrm{~K}$ is the most studied material in this system [6]. The $\mathrm{Nd}_{1.85} \mathrm{Ce}_{0.15} \mathrm{CuO}_{4-\mathrm{y}}$ compound has the same crystal structure as of $\mathrm{Nd}_{2} \mathrm{CuO}_{4}[\mathrm{I} / \mathrm{mmm}, \mathrm{a}=$ $3.945 \AA$ and $\mathrm{c}=12.17 \AA]$ but has slight variation in lattice parameters $[\mathrm{a}=3.945 \AA$ and $\mathrm{c}=12.076 \AA]$ due to the substitution of tetravalent $\mathrm{Ce}$ in the trivalent $\mathrm{Nd}$ lattice $[7,8]$. It is therefore of high interest to study the structural characteristics of a divalent dopant $\left(\mathrm{Sr}^{2+}\right)$ in $\mathrm{Nd}_{2} \mathrm{CuO}_{4}$ system and to assess influence on dielectric and magnetic properties of defective structures.

In the $\mathrm{Nd} / \mathrm{Ce}-\mathrm{Cu}-\mathrm{O}$ system, trivalent $\mathrm{Nd}^{3+}$ is replaced with tetravalent $\mathrm{Ce}^{4+}$ element which induces n-type superconductivity and crystallizes in two different crystal structures as mentioned earlier namely $\mathrm{T}^{\prime}$ and $\mathrm{T} / \mathrm{O}$. In the 
present work, Strontium doped $\mathrm{Nd}_{2} \mathrm{CuO}_{4}$ system has been prepared by substituting Strontium $\left(\mathrm{Sr}^{2+}\right)$ in Neodymium $\left(\mathrm{Nd}^{3+}\right)$ site inducing excess number of holes in the system which leads to $\mathrm{p}$-type superconductivity in the prepared compounds. Structure identification, surface morphology with composition analysis, magnetic properties, electron spin resonance and dielectric studies have been carried out and the results are discussed.

\section{Experimental Model}

\subsection{Sample Preparation}

The ceramic compounds $\mathrm{Nd}_{2-\mathrm{x}} \mathrm{Sr}_{\mathrm{x}} \mathrm{CuO}_{4}$ (where $\mathrm{x}=0.05$ to 0.2 ) have been prepared by carefully mixing stoichiometric quantities of $\mathrm{Nd}_{2} \mathrm{O}_{3} \quad\left(99.9 \%\right.$ purity), $\mathrm{SrCO}_{3}$ (99.99\% purity) and $\mathrm{CuO}$ (99.99\% purity) in an agate mortar with acetone as a mixing medium. Solid state reaction takes place during successive sintering sessions in alumina crucibles. The samples were precalcined initially at $850^{\circ} \mathrm{C}$ for 24 hours and the calcined samples were once again ground thoroughly prior to heating at $900^{\circ} \mathrm{C}$ for 24 hours. The final processing of sintering was done thrice at $925^{\circ} \mathrm{C}$ for 24 hours to obtain homogeneity and then slow cooled at a rate of $5^{\circ} \mathrm{C} /$ hour down to $300^{\circ} \mathrm{C}$. These hard sintered samples after final sintering were well ground and taken for characterization process.

\subsection{Powder X - Ray Diffraction}

The single phase formation of the compound was confirmed through powder XRD patterns which were recorded using PANalytical X'Pert PRO Diffractormeter with $\mathrm{CuK} \alpha 1$ radiation $(\lambda=1.54056 \AA)$. The diffraction data of the samples were collected with $0.02^{\circ} 2 \theta$ steps and $1 \mathrm{sec}$ count time per step for a $2 \theta$ range of $10^{\circ}$ to $80^{\circ}$.

\subsection{SEM and EDAX}

Micro structural surface morphology and elemental composition of the prepared compounds were carried out using Scanning Electron Microscope (SEM) added with Energy Dispersive X-ray Analysis (EDX) facility. In the present work, microscopic imaging and energy dispersive X-ray analysis (EDX) were simultaneously carried out using Hitachi S-3400N instrument. The surface of the pelletized compounds were focused with $5 \mathrm{kV}$ accelerating voltage under high vacuum condition.

\subsection{Magnetization Studies}

The variation in induced magnetic moment with respect to the applied magnetic field would be identified by $\mathrm{Vi}$ brating sample magnetometer (VSM) for the analysis of magnetic nature of the materials. In the present work, the magnetization experiment was carried out by employing LAKESHORE Vibrating Sample Magnetometer at room temperature $(300 \mathrm{~K})$ to categorize the magnetic property of all the prepared compounds.

\subsection{Electron Spin Resonance Studies}

Interaction of unpaired electron spins with respect to the external magnetic field yields the electronic nature of the source material utilized. Electron Spin Resonance (ESR) or Electron Paramagnetic Resonance (EPR) is a sophisticated spectroscopic technique that detects free radicals of inorganic complexes by which electronic structure and magnetic nature may be identified.

In the present work, ESR measurements were carried out using Varian E-4 spectrometer having X-band frequencies $(9.45 \mathrm{GHz})$. ESR spectra were recorded at room temperature and all the observations were performed with approximately $10 \mathrm{~mW}$ microwave power incident upon the sample cavity. The spectrometer was equipped with an electromagnet capable of producing a stable magnetic field from $0.001 \mathrm{~T}$ up to $0.8 \mathrm{~T}$ with the accuracy of $0.0001 \mathrm{~T}$. The spectrum is the first derivative microwave absorption with respect to field $(\mathrm{dP} / \mathrm{dH})$. For each sample, the $\mathrm{Hr}$ resonant magnetic field [9] and the value of peak-to-peak line-width $(\Delta \mathrm{Hpp})$ was computed as the difference between the extreme values $\mathrm{H}_{1}$ and $\mathrm{H}_{2}$ of the magnetic field (the maximum and minimum of the resonance curves, respectively). The resonant magnetic field $(\mathrm{Hr})$ was computed as $\left(\mathrm{H}_{1}+\mathrm{H}_{2}\right) / 2$. In order to make better comparative analysis the spectra were recorded by keeping the instrument settings same for all the samples.

\subsection{Dielectric Studies}

The study on dielectric constant with respect to the applied a.c frequency enumerates the nature of the atoms, ions and its bonding in the material. It is a measure of polarization in the medium. In the present work, HIOKI 3532-50 LCR HITESTER has been employed for the analysis of dielectric nature of the prepared compounds in pelletized form at room temperature condition with in the frequency range of $50 \mathrm{~Hz}$ to $5 \mathrm{MHz}$. Silver electrode pasting has been incorporated for to improve the surface conductivity of the prepared samples.

\section{Results and Discussion}

\subsection{Powder X-ray Diffraction Analysis}

The observed powder $\mathrm{X}$-ray diffraction patterns of the prepared polycrystalline samples $\mathrm{Nd}_{2} \mathrm{CuO}_{4}, \mathrm{Nd}_{1.95} \mathrm{Sr}_{0.05} \mathrm{CuO}_{4-\mathrm{y}}$, $\mathrm{Nd}_{1.9} \mathrm{Sr}_{0.1} \mathrm{CuO}_{4-\mathrm{y}}, \mathrm{Nd}_{1.85} \mathrm{Sr}_{0.15} \mathrm{CuO}_{4-\mathrm{y}}$ and $\mathrm{Nd}_{1.8} \mathrm{Sr}_{0.2} \mathrm{CuO}_{4-\mathrm{y}}$ are shown in Figure 1(a) which reveals the single phase formation of the prepared compounds. Lattice parameters of the compounds were calculated through AUTOX-93, a program for auto indexing reflections from multiphase polycrystals [10]. The comparison of lattice parameters observed for the prepared compounds with the parent compound is given in Table 1. The calculated unit cell parameters shows that the crystal structure of all the pre- 
pared compounds vary from the parent tetragonal structure to orthorhombic with least difference in magnitudes. Figures 1(b) and 1(c) show the shift in the higher intensity peaks of the prepared compounds with the formation of newer peaks (marked in * symbol) which reveals variation in the crystal system and lattice parameters of the prepared compounds. Due to the substitution of lower atomic radii element $(\mathrm{Sr}-2.45 \AA)$ in the higher atomic radii element site $(\mathrm{Nd}-2.64 \AA)$, the volume of the prepared compounds decreases with respect to the parent compound. Hence from the XRD patterns, it may be concluded that the Neodymium site is partly replaced with the Strontium atom.

\subsection{Surface Morphology and Elemental Analysis}

The surface morphology and crystallization nature of the samples were analyzed by scanning electron microscope images and the observed images for the prepared compounds are shown in Figure 2. In the present work, the pelletized samples annealed at $925^{\circ} \mathrm{C}$ were used for the surface morphology analysis.

The images of the inner portion of the pellets show that all the compounds have regular crystallites with the size in the micrometer range. The regular arrangement of particles reveals that the compounds were formed in well crystalline nature without clustering. Some amount of agglomeration of particles in the parent compound may be due to low melting point of the same as compared with Strontium substituted compounds. The elemental composition analysis confirms the presence of Strontium in the host matrix of $\mathrm{Nd}_{2} \mathrm{CuO}_{4}$ system with increasing concentrations in the samples. The decrease in the concentration of $\mathrm{Nd}$ reveals the replacement of trivalent Neodymium with divalent Strontium element.

\subsection{Magnetization Analysis}

The observed magnetization nature of the prepared compounds is shown in Figure 3. It is observed that the intensity of magnetization varies linearly with the applied magnetic field for all the prepared compounds which reveals the paramagnetic nature of the resultant

Table 1. Comparative statement of lattice parameters observed for parent and substituted compounds

\begin{tabular}{|c|c|c|c|c|c|}
\hline Compound & a $(\AA)$ & b $(\AA)$ & c $(\AA)$ & $\begin{array}{l}\text { Volum } \\
\text { e }\left(\AA^{3}\right)\end{array}$ & $\begin{array}{l}\text { Syst } \\
\text { em* }\end{array}$ \\
\hline $\mathrm{Nd}_{2} \mathrm{CuO}_{4}$ & 3.945 & 3.945 & 12.17 & 189.2 & $\mathrm{~T}$ \\
\hline $\begin{array}{c}\mathrm{Nd}_{1.95} \mathrm{Sr}_{0.05} \\
\mathrm{CuO}_{4-\mathrm{y}}\end{array}$ & $3.945(3)$ & $3.927(6)$ & $12.16(1)$ & 188.6 & $\mathrm{O}$ \\
\hline $\begin{array}{c}\mathrm{Nd}_{1.9} \mathrm{Sr}_{0.1} \\
\mathrm{CuO}_{4-\mathrm{y}}\end{array}$ & $3.826(5)$ & $3.943(3)$ & $12.19(1)$ & 184.1 & $\mathrm{O}$ \\
\hline $\begin{array}{c}\mathrm{Nd}_{1.85} \mathrm{Sr}_{0.15} \\
\mathrm{CuO}_{4-\mathrm{y}}\end{array}$ & $3.832(3)$ & $3.946(2)$ & $12.18(6)$ & 184.3 & $\mathrm{O}$ \\
\hline $\begin{array}{c}\mathrm{Nd}_{1.8} \mathrm{Sr}_{0.2} \\
\mathrm{CuO}_{4-\mathrm{y}}\end{array}$ & $3.897(6)$ & $3.901(6)$ & $12.09(1)$ & 183.9 & $\mathrm{O}$ \\
\hline
\end{tabular}

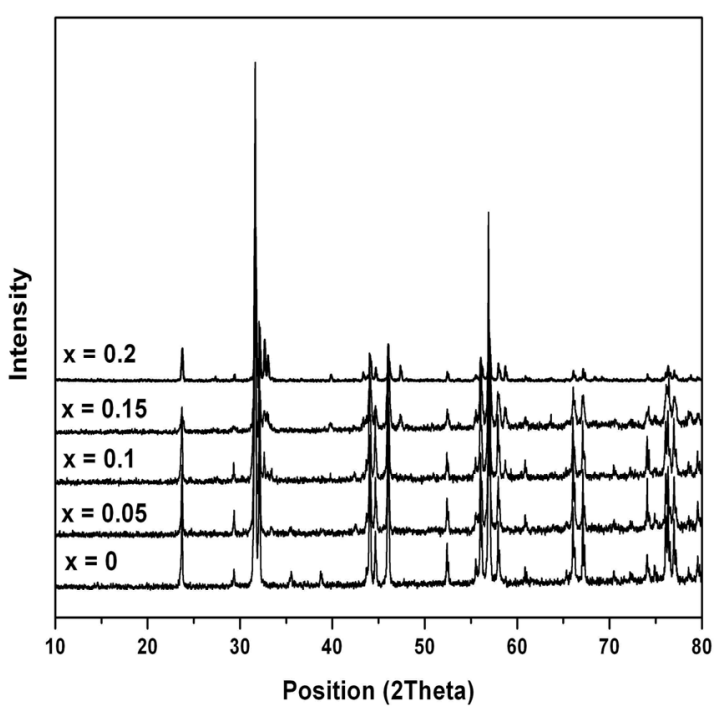

(a)

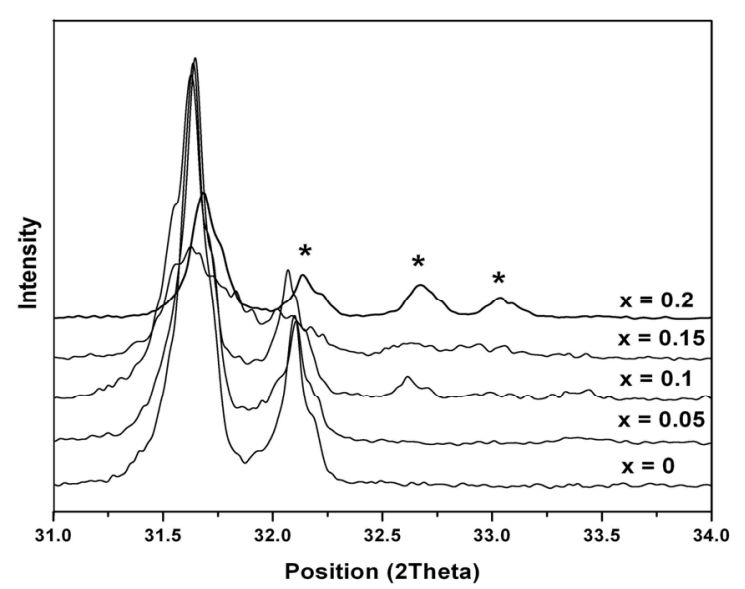

(b)

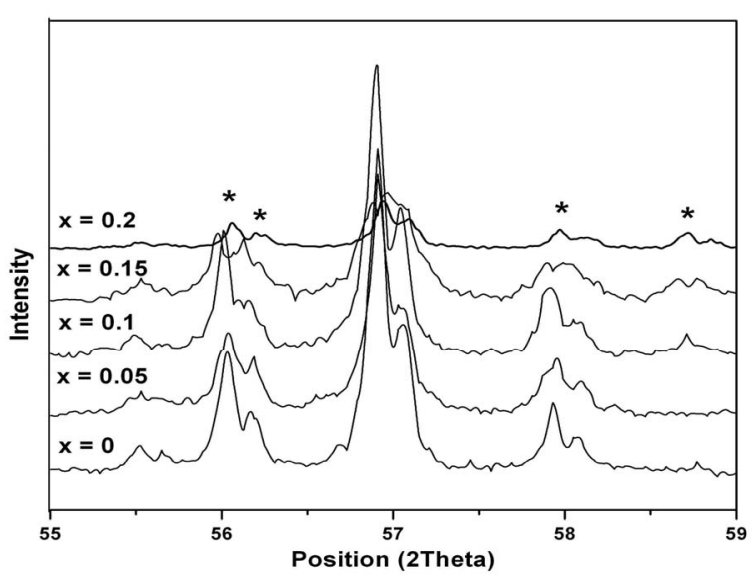

(c)

Figure 1. (a) Powder X-Ray Diffraction patterns of the prepared compounds; (b) Comparison of diffraction patterns of the major intensity peak; (c) Comparison of diffraction patterns of the $50 \%$ intensity peak 


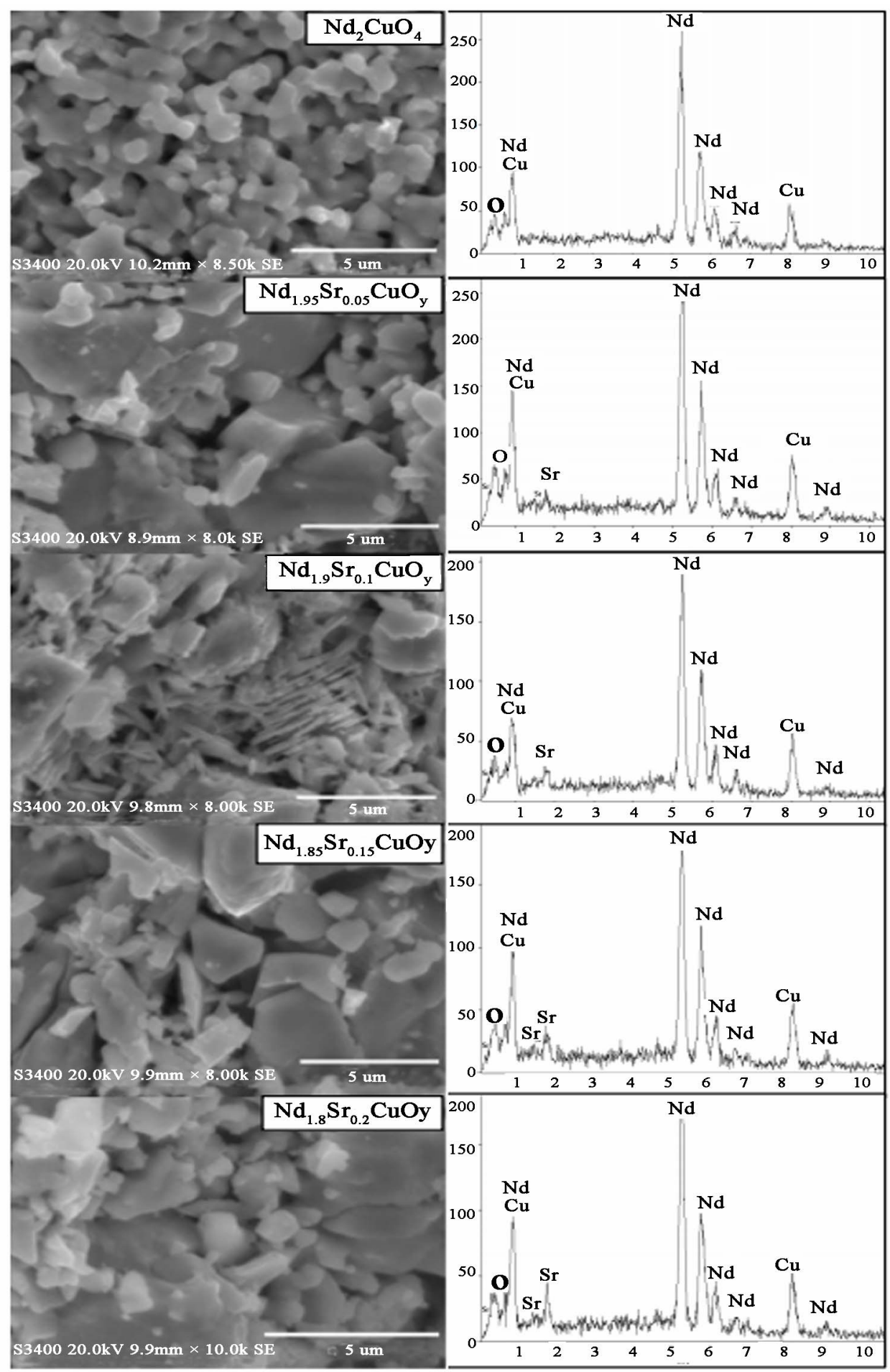

Figure 2. Surface Morphology and EDX spectrum of the prepared compounds

compounds $[11,12]$. In the earlier reports, it is identified that the parent compound $\mathrm{Nd}_{2} \mathrm{CuO}_{4}$ exhibits paramagnetic nature [13] and the same is obtained for both parent and prepared compounds now. The net magnetic moment attained by the prepared compounds is in very minimal level (memu/g) which exhibits the least possible magnetization of the prepared compounds and not more significant changes on the magnetization nature are observed on substitution of Strontium in Neodymium site. Hence it is confirmed that all the prepared compounds 
are exhibiting paramagnetic nature at room temperature.

\subsection{ESR Analysis}

Figures 4(a) and 4(b) show the room-temperature X-band $(9.45 \mathrm{GHz})$ ESR spectra of $\mathrm{Nd}_{2} \mathrm{CuO}_{4}$ samples with four different concentrations of Strontium. The room temperature ESR spectrum shows an intense resonance signal for the $\mathrm{Nd}_{2} \mathrm{CuO}_{4}$ and $\mathrm{Nd}_{1.95} \mathrm{Sr}_{0.05} \mathrm{CuO}_{4-\mathrm{y}}$ compounds with both peak to peak line width $\left(\Delta \mathrm{H}_{\mathrm{PP}}\right)$ and resonance field $\left(\mathrm{H}_{\mathrm{r}}\right)$ that vary depending on the samples. The line width of the samples was calculated as $0.2852 \mathrm{~T}$ and $0.2892 \mathrm{~T}$ for the samples $\mathrm{Nd}_{2} \mathrm{CuO}_{4}$ and $\mathrm{Nd}_{1.95} \mathrm{Sr}_{0.05} \mathrm{CuO}_{4-\mathrm{y}}$ respectively. The Lande factor of the samples with sharp resonance is calculated and the values are 2.37 and 2.33 for $\mathrm{Nd}_{2} \mathrm{CuO}_{4}$ and $\mathrm{Nd}_{1.95} \mathrm{Sr}_{0.05} \mathrm{CuO}_{4-\mathrm{y}}$ respectively. The samples with an effective $\mathrm{g}$ value of around 2 shows the paramagnetic behavior which has a good agreement with results obtained from magnetic measurements ( $\mathrm{M}$ vs $\mathrm{H}$ curves). Other three samples with higher Strontium concentration show no resonance absorption bands. But the intensity remains constant up to 3000 Gauss and then decreases exponentially. This condition implies the least magnetization nature of the prepared samples. The variation in $\Delta \mathrm{H}_{\mathrm{PP}}$ with respect to Strontium substitution in the parent system may be due to the presence of inhomogeneities and differences in the chemical composition or in the oxygen stoichiometry $[14,15]$.

\subsection{Dielectric Analysis}

The dielectric studies have been carried out for the parent and the strontium substituted compounds. This study gives experimental values such as capacitance at parallel and dielectric loss for the frequency range between 50 $\mathrm{Hz}$ to $5 \mathrm{MHz}$ at the room temperature for the analysis of

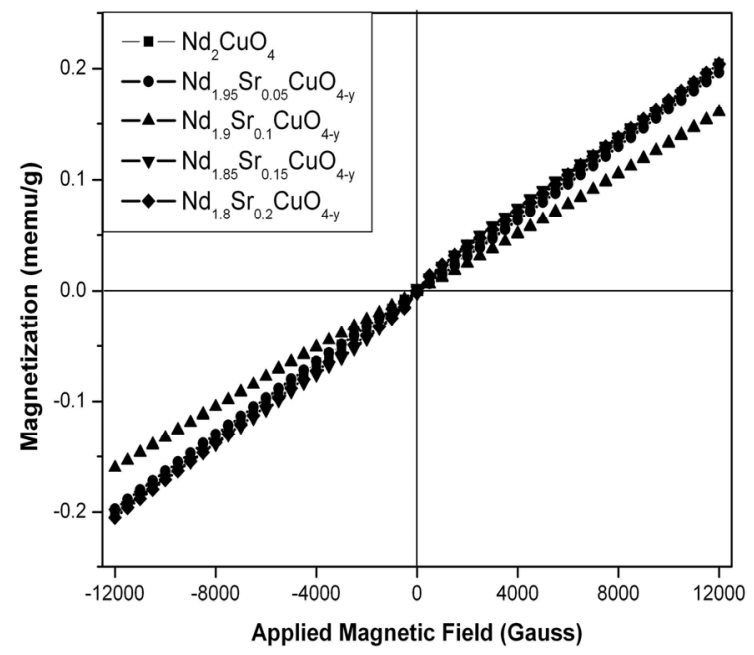

Figure 3. Magnetization nature of the prepared compounds

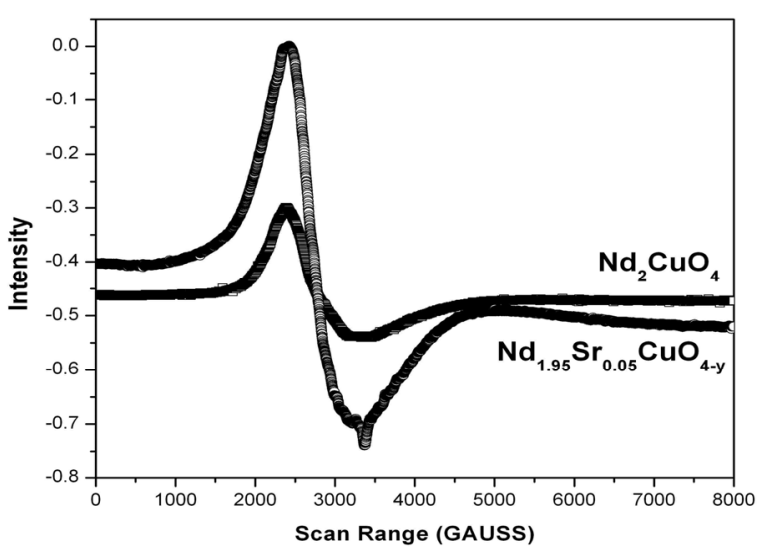

(a)

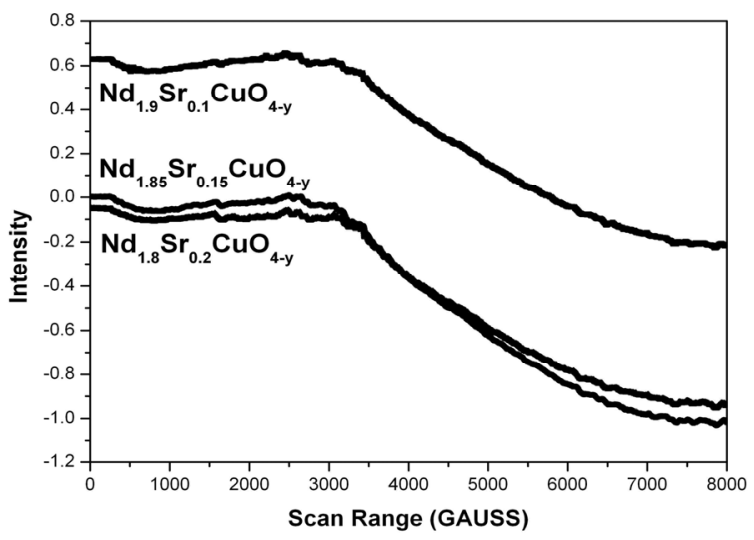

(b)

Figure 4. Electron Spin Resonance nature of the prepared compounds

dielectric behaviour of the prepared compounds. The dielectric constant was calculated from the formula; $\boldsymbol{\varepsilon}_{\mathbf{r}}=$ $\left(\mathbf{C}_{\mathbf{P}} \mathbf{d}\right) /\left(\boldsymbol{\varepsilon}_{\mathbf{0}} \mathbf{A}\right)$ where $\mathrm{C}_{\mathrm{P}}$ is the capacitance in parallel $(\mathrm{F})$, $\mathrm{d}$ is the thickness of the pellet $(\mathrm{m})$ and $\mathrm{A}$ is the cross sectional area of the pellet $\left(\mathrm{m}^{2}\right)$.

The plots of logf versus dielectric constant and dielectric loss have been drawn (Figures 5(a) and 5(b)). It is observed that the parent compound has the highest dielectric constant values than the strontium substituted compounds. It may due to the fact of introduction of holes in the host $\mathrm{Nd}_{2} \mathrm{CuO}_{4}$ system. At the same time, increasing order of dielectric constant on higher doping concentration of strontium shows the improved surface transport properties. The high value of dielectric constant at low frequencies may be associated with the establishment of polarizations namely; space charge, orientational, electronic and ionic polarization. The low value of dielectric constant at higher frequencies may be due to the loss of significance of these polarizations gradually. The 


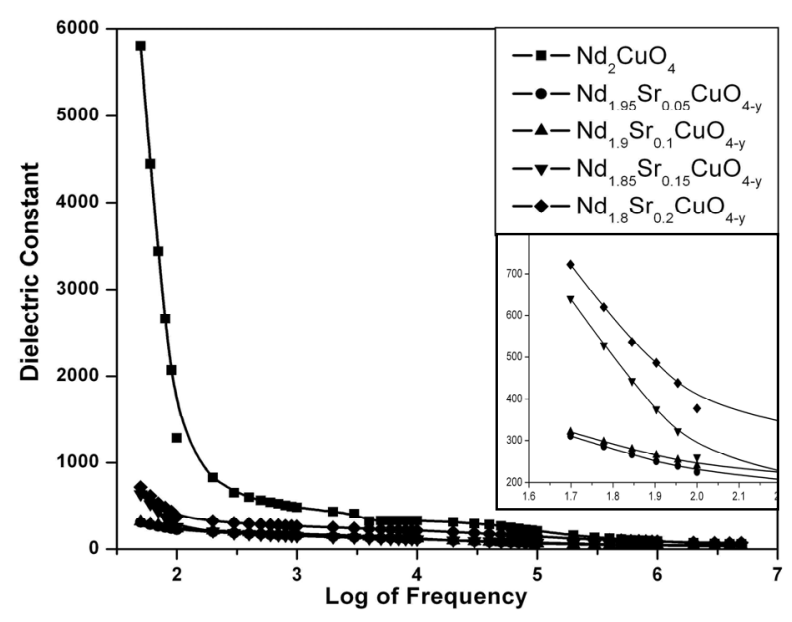

(a)

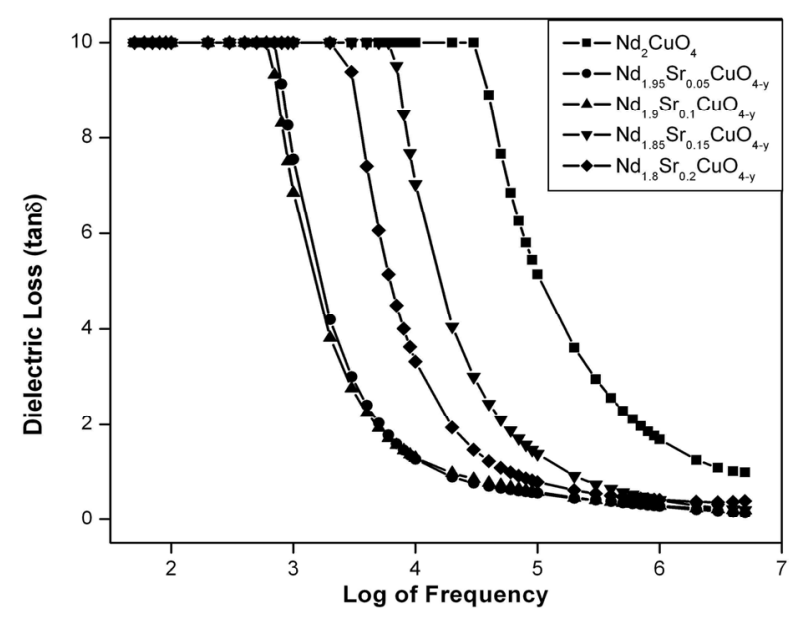

(b)

Figure 5. (a) Plots of dielectric constant of the prepared compounds; (b) Plots of dielectric loss of the prepared compounds

frequency dependence of dielectric loss exhibits interesting results. At the lower frequencies, the dielectric loss reaches the instrumental saturation value $(\tan \delta=$ 9.9999) but at higher frequencies the value drops down from this saturation drastically. The low value of dielectric loss at higher frequencies implies that all the samples possess superior optical quality and shows the possibility of using the prepared compounds for high frequency applications.

\section{Conclusions}

In the present work, the effect of partial substitution on Neodymium site in $\mathrm{Nd}_{2} \mathrm{CuO}_{4}$ system with Strontium was carried out. The ceramic compounds $\mathrm{Nd}_{2} \mathrm{CuO}_{4}$, $\mathrm{Nd}_{1.95} \mathrm{Sr}_{0.05} \mathrm{CuO}_{4-\mathrm{y}}, \quad \mathrm{Nd}_{1.9} \mathrm{Sr}_{0.1} \mathrm{CuO}_{4-\mathrm{y}}, \quad \mathrm{Nd}_{1.85} \mathrm{Sr}_{0.15} \mathrm{CuO}_{4-\mathrm{y}}$ and $\mathrm{Nd}_{1.8} \mathrm{Sr}_{0.2} \mathrm{CuO}_{4-\mathrm{y}}$ were prepared by solid state reaction technique with high purity chemicals. Structural characterization was carried out by using powder X-ray diffraction technique and it was found that the parent compound crystallized in tetragonal structure and the other newly prepared compounds were crystallized in orthorhombic structure. Surface morphology confirms the high crystalline nature of the prepared compounds whereas increasing order of Strontium in the $\mathrm{Nd}_{2} \mathrm{CuO}_{4}$ system is confirmed through elemental compositions using energy dispersive analysis. The magnetic nature of the compounds was identified with vibrating sample magnetometer and it was found that both parent and prepared compounds exhibit paramagnetic nature at room temperature. Increasing concentration of Strontium in the $\mathrm{Nd}_{2} \mathrm{CuO}_{4}$ system results in the decrease in net magnetization of all the prepared compounds. The same results are also identified in the electron paramagnetic resonance studies through the least resonance absorption intensity of the parent and the prepared compounds. Increase in dielectric constant on higher doping concentration confirms the incorporation of Strontium in the $\mathrm{Nd}_{2} \mathrm{CuO}_{4}$ system and the least value of dielectric loss at higher frequencies confirms the improved surface transport properties of the prepared compounds.

\section{REFERENCES}

[1] Y. Tokura, H. Takagi and S. Uchida,"A Superconducting Copper Oxide Compound with Electrons as the Charge Carriers," Nature, Vol. 337, 1989, pp. 345-347.

[2] H. Takagi, S. Uchida and Y. Tokura, "Superconductivity Produced by Electron Doping in $\mathrm{CuO}_{2}$-Layered ComPounds," Physical Review Letters, Vol. 62, No. 10, 1989, pp. 1197-1200.

[3] H. Muller-Buschbaum and W. Z. Wollschlager, "Über Ternäre Oxocuprate. VII. Zur Kristallstruktur von $\mathrm{Nd}_{2} \mathrm{CuO}_{4}$," Zeitschrift für Anorganische und Allgemeine Chemie, Vol. 414, 1975, pp. 76-80.

[4] H. Muller-Buschbaum and W.Z. Wollschlager, "Über Oxocuprate. XV Zur Kristallstruktur von Seltenerd Metal Oxocupraten: $\mathrm{La}_{2} \mathrm{CuO}_{4}, \mathrm{Gd}_{2} \mathrm{CuO}_{4}$," Zeitschrift für Anorganische und Allgemeine Chemie, Vol. 428, 1975, pp. 120124.

[5] T. V. Ramakrishnan and C. N. R. Rao, "Superconductivity Today-An Elementary Introduction," 2nd Edition, Universites Press (India), Hyderabad, 1999, pp. 26-54.

[6] G. H. Kwei, S. W. Cheong, Z. Fisk, F. H. Garzon, J. A. Goldstone and J. D. Thompson, "Structure and Oxygen Stoichiometry for the Electron-Doped Cuprate Superconductor $\mathrm{Nd}_{1.85} \mathrm{Ce}_{0.15} \mathrm{CuO}_{4-\mathrm{d}}$," Physical Review B, Vol. 40, 1989, pp. 9370-9373.

[7] X. Zhang, C. R. A. Catlow, S. C. Parker and A. Wall, "Simulation Study of Pressure-Induced Structural Changes in $\mathrm{La}_{2} \mathrm{CuO}_{4}$ and in $\mathrm{La}_{1.83} \mathrm{Sr}_{0.17} \mathrm{CuO}_{4}$," Journal of Physics 
and Chemistry of Solids, Vol. 53, No. 6, 1992, pp. 761-770.

[8] E. F. Paulus, I. Yehia, H. Fuess, J. Rodriguez, T. Vogt, J. Ströbel, M. Klauda and G. Saemann-Ischenko, "Crystal Structure Refinement of $\mathrm{Nd}_{2-x} \mathrm{Ce}_{x} \mathrm{CuO}_{r m 4}(x=0.05-0.30)$ by X-Ray $(295 \mathrm{~K})$ and Neutron (1.5 K) Powder Diffraction," Solid State Communications, Vol. 73, No. 11, 1990, pp. 791-795.

[9] J. F. Raber, "Experimental Methods in Polymer Chemistry-Physical Principal and Applications," John Wiley, New York, 1980, pp. 332-352.

[10] V. B. Zlokazov, "AUTOX-A Program for Autoindexing Reflections from Multiphase Polycrystals," Computer Physics Communications, Vol. 85, No. 3, 1995, pp. 415-422.

[11] O. D. Jayakumar, I. K. Gopalakrishnan and S. K. Kulshreshtha, "The Structural and Magnetization Studies of Co-Doped $\mathrm{ZnO}$ Co-Doped with $\mathrm{Cu}$ : Synthesized by CoPrecipitation Method," Journal of Materials Chemistry, Vol. 15, No. 34, 2005, pp. 3514-3518.
[12] O. D. Jayakumar, I. K Gopalakrishnan, and S. K. Kulshreshtha, "On the Room Temperature Ferromagnetism of Mn Doped ZnO,” Physica B, Vol. 381, No. 1-2, 2006, pp. 194-198.

[13] H. Samata, T. Komiyama, S. Tsuboi, Y. Nagata, T. Uchid, M. Ohtsuk and M. Der Lan, "Electrochemical Crystal Growth of Superconducting Cuprates," Journal of Physics and Chemistry of Solids, Vol. 58, No. 10, 1997, pp. 15471552.

[14] J. A. Olarte, L. C. Moreno and A. Mariño, "Susceptibility and EPR Studies of $\mathrm{LaMn}_{x-1} \mathrm{Co}_{x} \mathrm{O}_{3}$ Synthesized by Citrate Precursor Method," Microelectronics Journal, Vol. 39, No. 11, 2008, pp. 1245-1247.

[15] S. Angappane, M. Pattabiraman, G. Rangarajan, K. Sethupathi, B. Varghese and V. S. Sastry, "ESR Study of Spin-Lattice Correlated Clusters in Single Crystalline $\mathrm{Nd}_{0.7} \mathrm{Sr}_{0.3} \mathrm{MnO}_{3}$," Journal of Physics: Condensed Matter, Vol. 19, 2007, pp. (036207)1-16. 